\title{
Système National de Recherche et d'Innovation en Afrique : le cas du Cameroun
}

Article · May 2017

CITATIONS

0

5 authors, including:
READS

5

\section{Temple Ludovic}

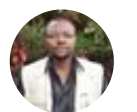

Cirad - La recherche agronomique pour le dé...

145 PUBLICATIONS 510 CITATIONS

SEE PROFILE
Guillaume Hensel Fongang Fouepe

Université de Dschang

42 PUBLICATIONS 3 CITATIONS

SEE PROFILE

Some of the authors of this publication are also working on these related projects:

Project

AgriSpin: Focusing innovation support services conceptual grounds \& research framework View project

Mutations dans l'éducation, la formation, la vulgarisation et le conseil agricoles: le cas du Cameroun Project 


\section{SYSTÈME NATIONAL DE RECHERCHE ET D'INNOVATION EN AFRIQUE : LE CAS DU CAMEROUN \\ Ludovic Temple, Syndhia Mathé, Nadine Machicou Ndzesop, Guillaume Hensel \\ Fongang Fouepe, Michel Ndoumbe Nkeng}

De Boeck Supérieur | «Innovations »

2017/0 Prépublication | pages art14_I à art14_XXVI

ISSN 1267-4982

Article disponible en ligne à l'adresse :

http://www.cairn.info/revue-innovations-2017-0-page-art14_I.htm

\section{Pour citer cet article :}

Ludovic Temple et al., "Système National de Recherche et d'Innovation en Afrique : le cas du Cameroun », Innovations 2017/0 (Prépublication), p. art14_I-art14_XXVI.

Distribution électronique Cairn.info pour De Boeck Supérieur.

(C) De Boeck Supérieur. Tous droits réservés pour tous pays.

La reproduction ou représentation de cet article, notamment par photocopie, n'est autorisée que dans les limites des conditions générales d'utilisation du site ou, le cas échéant, des conditions générales de la licence souscrite par votre établissement. Toute autre reproduction ou représentation, en tout ou partie, sous quelque forme et de quelque manière que ce soit, est interdite sauf accord préalable et écrit de l'éditeur, en dehors des cas prévus par la législation en vigueur en France. Il est précisé que son stockage dans une base de données est également interdit. 


\title{
SYSTĖME NATIONAL DE RECHERCHE ET D'INNOVATION EN AFRIQUE : LE CAS DU CAMEROUN ${ }^{1}$
}

\author{
Ludovic TEMPLE \\ Centre de Coopération Internationale en Recherche Agronomique \\ pour le Développement \\ UMR Innovation, France \\ ludovic.temple@cirad.fr \\ Nadine MACHICOU NDZESOP \\ Université de Yaoundé 2, Cameroun \\ nadngameni@yahoo.fr \\ Guillaume Hensel FONGANG FOUEPE \\ Université de Dschang, Cameroun \\ guillaumefongang@yahoo.fr \\ Michel NDOUMBE NKENG
}

Institut de Recherche Agricole pour le Développement, Cameroun ndoumbe.nkeng@yahoo.com Syndhia MATHÉ Centre de coopération Internationale en Recherche Agronomique pour le Développement

UMR Innovation International Institute of Tropical-Agriculture, Cameroun syndhia.mathe@cirad.fr

\section{RÉSUMÉ}

La transition sociodémographique des pays du sud structure les marchés intérieurs et crée des opportunités de croissance économique. En mobilisant le concept de Système National de Recherche et d'Innovation, nous testons en quoi il améliore la connaissance des conditions institutionnelles qui mobilisent les résultats de la recherche dans les processus d'innovation. Une première partie discute de l'adaptation de la notion de Système National

1. Nous remercions les référés anonymes qui par leurs commentaires ont enrichi et solidifié le texte initial. 
d'Innovation aux pays du Sud. Une seconde partie caractérise les conditions historiques d'émergence des institutions de recherche au Cameroun. Une troisième partie analyse en quoi la structure de la production scientifique depuis 1991 répond aux besoins d'innovations du secteur agricole et alimentaire. La quatrième partie explicite les conditions d'utilisation de cette production par les sous-systèmes de l'intermédiation et de l'entrepreneuriat. Les résultats interrogent comment l'usage de la notion de SNRI peut devenir un élément d'orientation des politiques de recherche et d'innovation finalisées par le développement.

Mots-clés : Système National d'Innovation, Cameroun, Politique d'innovation et de recherche, Développement, Secteur agricole et alimentaire

Codes JEL : 03, 032, 038, D83, H5

\section{ABSTRACT}

\section{National System of Research and Innovation in Africa: The Case of Cameroon}

The demographic transition of the Southern countries structure domestic markets and creates opportunities for economic growth. Drawing on the concept of National System of Research and Innovation, we test how it improves knowledge on the institutional conditions that mobilize the results of research in innovation processes. The first part discusses the adaptation of the concept of National System of Innovation in the Southern countries. A second part characterizes the conditions for emergence of research institutions in Cameroon. A third part analyzes how the structure of the scientific production has, since 1991, met the needs of innovation in the agricultural and food sector. The fourth part explains the conditions of use of this production by the subsystems of intermediation and entrepreneurship. The results discuss how the use of the notion of NSRI can become an element used in the orientation of research and innovation policies.

Keywords: National Innovation System, Cameroon, Innovation and Research Policies, Development

JEL Codes: 03, 032, 038, D83, H5

Le renouvellement des politiques de recherche et leur articulation aux processus d'innovation (Laredo, Mustar, 2003) ouvrent plusieurs questions sur l'évolution des structures de financement de la recherche publique, la gouvernance des interfaces entre la recherche, l'entrepreneuriat, et les méthodes qui évaluent les causalités entre l'activité de recherche et le développement (Bornman, 2013 ; Temple et al., 2016). L'investissement dans la recherche est souvent considéré comme un levier de l'innovation pour réduire les inégalités technologiques et les disparités de développement. Dans la recherche agronomique par exemple, de nombreuses analyses économiques calculent une rentabilité financière élevée des investissements (Maredia, Raitzer, 2010). Leurs conditions de réalisation sont cependant tributaires de la spécificité des environnements institutionnels nationaux et sectoriels qui sont fragilisés dans les pays en développement particulièrement en Afrique 
(Nyemeck, Nkamleu, 2006). La notion de Système National de Recherche est au cœur des usages pour analyser ces environnements (Gaillard, 2003). Cette étude interroge l'efficacité de la recherche dans sa contribution aux processus d'innovations. Elle se focalise en cela sur la connexion (relation) entre la recherche nationale et les demandes entrepreneuriales du secteur productif. L'hypothèse posée au regard de la littérature est que cette connexion est le levier des causalités qui lient l'activité de recherche et les mécanismes de développement qui répondent aux attentes sociétales. Pour certains auteurs cette connexion doit renforcer les capacités d'innovation et d'apprentissage pour mobiliser les connaissances scientifiques et technologiques que proposent les pays industriels (Casadella et al., 2015) et qui favorisent les transferts technologiques. Cette proposition suppose cependant que ces connaissances élaborées dans des pays industriels soient adaptées à la spécificité des structures productives et aux potentialités que proposent les ressources (humaines et matérielles) des sociétés locales.

L'Afrique étant une réalité géopolitique hétérogène, nous focalisons le propos sur le Cameroun, considéré comme une "économie témoin » d'un certain nombre d'indicateurs régionaux. Situé au creux du golfe de Guinée, ce pays de 23 millions d'habitants ${ }^{2}$ est dans la tranche basse des pays à revenu intermédiaire. En symétrie avec d'autres pays africains, le taux de croissance du PIB croît autour de $5 \%$ par an depuis 2005. Cette croissance ne permet pas, dans les conditions de sa répartition de diminuer la pauvreté qui affecte $40 \%$ de la population depuis 10 ans. Plus de $40 \%$ de cette population a moins de 20 ans (Minsat et al., 2015). La croissance rapide de la population active estimée à 7,2 millions en 2009 densifie les flux migratoires vers les villes. Une conséquence est l'augmentation de la demande intérieure marchande de biens (produits alimentaires, bâtiment, etc.) et services (éducation, santé, etc.) qui constitue une opportunité pour le secteur des entreprises et un levier potentiel d'accélération majeure de la croissance économique.

Le Cameroun a investi dans l'éducation et la recherche depuis les années 1970. De 2100 enseignants chercheurs en 1999 (Arvanitis et al., 2000 ; Waast et al., 2001), il en compte plus de 6300 aujourd'hui. Avec 300 chercheurs par million d'habitants ${ }^{3}$. Ce pays est cinquième à l'échelle de l'Afrique sub-saharienne après l'Égypte, le Maroc, le Sénégal, et l'Afrique du sud (African Union New Partenership for Africa's Development, 2014). Le ratio entre l'investissement dans la recherche et le PIB en valeur se situe autour de 0,3 dans la moyenne des pays moins avancés, mais inférieur à celui des pays émergents (autour de 0,8).

2. Estimation 2014, Institut National de la Statistique du Cameroun.

3. 886 chercheurs par million d'habitants dans les pays émergents. 
Nous proposons à partir de ce pays d'étudier en quoi les structures d'interactions entre les institutions de recherche et le secteur entrepreneurial mobilisent les connaissances scientifiques issues des investissements nationaux de recherche comme levier d'action dans le secteur entrepreneurial. En l'absence d'outils spécifiques aux contextes des pays en développement, nous mobilisons la notion de Système d'Innovation (Touzard et al., 2015) qui permet d'analyser les dynamiques d'innovation à l'échelle d'un territoire national. Nous préciserons dans quelle mesure cette notion structure l'existence d'un Système National de Recherche et d'Innovation (SNRI).

La démonstration se fera en quatre temps. Le premier interroge la pertinence de la mobilisation du concept de Système National d'Innovation (SNI) comme outil d'analyse des politiques de recherche et d'innovation dans le contexte des pays en développement (Mytelka, 2003). Le deuxième temps référence les conditions historiques d'émergence des institutions de recherche pour expliquer les structures actuelles du système. Le troisième temps étudie les caractéristiques de la production scientifique du pays depuis 1991. Enfin, nous interrogeons les conditions d'usages de cette production au sein des institutions de l'intermédiation en fonction des structures industrielles polarisées par le secteur agricole, pivot de l'industrialisation actuelle.

\section{CADRE CONCEPTUEL ET MÉTHODOLOGIQUE}

\section{Du Système National d'Innovation [SNI] au Système National de Recherche et d'Innovation?}

De manière conventionnelle, un système d'innovation est composé d'un ensemble d'institutions, d'organisations, et de réseaux d'acteurs qui interagissent pour favoriser le changement technologique dans un espace géographique ou institutionnel structuré par des entreprises ou le développement d'une technologie (Touzard et al., 2015). L'usage de la notion de Système National d'Innovation (SNI) est croissant depuis la fin des années 1980 au sein des institutions internationales de développement (OCDE, Union Européenne, Banque Mondiale, FAO). Le premier usage a été appliqué au cadre d'un territoire national (Lundvall, Lemapages, 2014 ; Gallaud, 2014) susceptible de structurer des ressources institutionnelles, entrepreneuriales et scientifiques spécifiques dont la mise en complémentarité organisationnelle génère l'innovation (Freeman, 2002). L'application de cet usage dans les pays les moins avancés ou à revenus intermédiaires donne lieu à deux critiques principales.

La première souligne en quoi respectivement la privatisation des investissements de recherche (Carlson, 2006), la fragilisation des politiques 
publiques par les programmes d'ajustements structurels entre les années 1980 et 2000 (Uzunidis, Laperche, 2011) et la spécificité sectorielle ; fragilisent la capacité du territoire national à « mettre en système » les ressources mobilisables pour l'innovation. Cette critique structure d'autres échelles d'analyses en termes de systèmes régionaux d'innovation rejoignant les travaux sur les clusters, les districts, les systèmes locaux de production et en termes de Systèmes Sectoriels d'Innovation (Malerba, 2002).

La seconde critique en relation avec la fragilité du secteur industriel des pays qualifiés de «non hégémoniques " porte sur la difficulté des pays non industriels à bénéficier des ressources que génèrent les investissements publics nationaux de recherche. Ainsi les activités de recherche y seraient fragmentées, gouvernées par les normes, les financements et les opportunités des pays industriels. Elles participeraient plus à la production de progrès scientifiques globalisés ou produisent des ressources humaines mobilisables par de grandes entreprises.

Ces deux critiques appellent au renforcement et l'adaptation des politiques de recherche et d'innovation au contexte des pays à revenus intermédiaires ou moins avancés. Elles conduisent à tester la pertinence du concept de Système National de Recherche et d'Innovation par une triple analyse :

i) contextuelle sur les conditions d'économie politique d'émergence des institutions de recherche. Il s'agit de comprendre le landscape macro-institutionnel (Geels, 2004);

ii) actorielle sur la caractérisation des parties prenantes du système d'innovation et de recherche, de leurs pouvoirs, leurs intérêts et incitations au changement ;

iii) fonctionnelle sur les interfaces entre les organisations qui portent une activité de recherche et celles qui mettent en œuvre les connaissances et résultats de recherche dans les processus d'innovation.

\section{Collecte des données et des informations utilisées}

La collecte des données est en partie générée par une expertise répondant à la demande du Ministère de la Recherche Scientifique et de l'Innovation du Cameroun (MINRESI). Cela avec l'objectif de mobiliser la recherche comme levier pour une transition vers l'émergence de l'économie camerounaise (Bisson et al., 2014). Elle utilise plusieurs sources de données :

- plus de 66 entretiens en face-à-face avec des représentants d'institutions de recherche, d'entreprises sur la base de guides d'entretien semidirectifs (cf. Tableau 1); 
- une étude bibliométrique (7 400 articles) sur les tendances et la structure de la production scientifique publiée au Cameroun: données du Web of ScienceTM. Core Collection: Citation Indexes (SCI-Expanded, SSCI, A\&HCI) produites parThomson Reuters ;

- trois ateliers de concertation des parties prenantes (70 participants) concernant respectivement les :

- instituts de recherche publique,

- acteurs de l'intermédiation des résultats de recherche (Organisations Professionnelles, ONG),

- industries du secteur agro-chimique phytosanitaire et semencier.

Les résultats de ces ateliers ont été synthétisés par l'élaboration de matrices identifiant les Atouts, Faiblesses, Opportunités et Menaces qui caractérisent la recherche publique (Bisson et al., 2014).

\section{Tableau 1 - Répartition des entretiens réalisés par catégories d'institution}

\begin{tabular}{|l|c|}
\hline Institutions & Effectif \\
\hline Universités & 20 \\
\hline Instituts du MINRESI & 20 \\
\hline Structures d'intermédiation (CICC, GICAM, SAILD, OP, Horticam, CCI, ONG] & 16 \\
\hline Entreprises secteur agro-alimentaire & 10 \\
\hline Total & 66 \\
\hline
\end{tabular}

Source : Enquêtes auteurs, 2014

Les enquêtes réalisées soulignent toutes le manque d'interaction entre les composantes universitaires et les instituts du dispositif de recherche. Ces dysfonctionnements étant aggravés par la différence de statut du chercheur (promotion, âge de la retraite...) et les conditions d'accès aux fonds publics de recherche. Dans un contexte de faiblesse des infrastructures expérimentales mobilisables, cette déconnexion ne permet pas ou peu de mutualiser l'usage pourtant possible des dispositifs d'expérimentation mobilisables (laboratoires et équipements).

La connaissance des liens entre les sous-systèmes : recherche, entrepreneurial et d'intermédiation est peu explicitée par les travaux mobilisables au Cameroun. Nous proposons d'y contribuer dans le secteur agricole et alimentaire qui polarise l'histoire de la recherche scientifique du pays et qui concentre près de $50 \%$ de la population active et environ $30 \%$ du PIB. Pour cela nous analysons en quoi la production scientifique est mobilisée ou mobilisable au sein des deux sous-systèmes entrepreneurial et d'intermédiation qui structurent le SNRI. 


\section{CONDITIONS D'ÉMERGENCE DU SYSTÈME NATIONAL D'INNOVATION ET DE RECHERCHE}

\section{Racines du système national de recherche scientifique du Cameroun}

L'encadrement de la recherche et de l'innovation trouve sa source dans une bonne compréhension de l'histoire institutionnelle de la recherche scientifique (Machikou, 2014).

Pendant la période coloniale allemande qui débute en 1889, l'activité de recherche est portée par des individus rattachés à différentes institutions : église, armée, universités ${ }^{4}$. C'est au lendemain de la première guerre mondiale, sous la tutelle française que la recherche se coordonne par l'installation des Services de l'Agriculture du Haut-Commissariat de la République du Cameroun. Des stations expérimentales voient le jour à Dschang en 1925 (café arabica et quinquina), à Ngaoundéré en 1930 (productions animales), à Bambui en 1933 (cultures vivrières), à Ebolowa en 1938 (café robusta et vivrier), à Nkongsamba (sols) et à Maroua (arachide et traction animale). La recherche s'institutionnalise au niveau national en 1935 par la Société d'Études Camerounaises.

Après la deuxième Guerre Mondiale, les premiers instituts français spécialisés dans la recherche agricole coloniale s'installent au Cameroun: l'Institut Français des Fruits et Agrumes Coloniaux (IFAC), à Njombé en 1944 ; l'Institut de Recherche pour les Huiles et Oléagineux (IRHO) à Dibamba en 1948 ; l'Institut d'Élevage et de Médecine Vétérinaire des pays Tropicaux (IEMVT) à Wakwa en 1955 et l'Institut de recherches du coton et des textiles exotiques en 1957. Dans la partie sous administration britannique, les stations expérimentales sont créées par des entreprises coloniales comme la Cameroon Development Corporation (CDC) respectivement en 1951 (Barombi-Kang) et en 1954 (Ekona). Sous la période postcoloniale, l'institutionnalisation du système national de recherche scientifique et technique reste instable. En 1961, les premiers investissements dans l'enseignement supérieur ont lieu et se concrétisent par la création de l'Université de Yaoundé suivie des Centres Universitaires de Douala, Ngaoundéré et Dschang. Il est créé l'École Nationale Supérieure Agronomique (ENSA) en 1977 avec l'appui de la coopération américaine ${ }^{5}$. Jusque dans les années 1970, le système national de la recherche scientifique et technique est

4. Création des jardins d'essais d'Edea et d'Akonolinga.

5. En 1988, l'ENSA et l'Institut des Techniques Agricoles (ITA) fusionnent pour devenir l'Institut National de Développement Rural (INADER). En 1993 est créée l'Université de Dschang. 
dominé par des instituts français et américains. L'Office National de la Recherche Scientifique et Technique (ONAREST), qui avait été mis sur pied en 1965 ne devient effectif qu'en 1974. Il devient l'organe scientifique du gouvernement et met sur pied 9 instituts de recherche dont principalement : le Centre National de Recherche Agronomique en 1972 (Ekona), et l'Institut de Recherches Médicales et d'Études des Plantes Médicinales en 1974 (IMPM). Les problèmes de cohérence de la politique de recherche entre les universités et les instituts conduisent à la création d'un Conseil de l'Enseignement Supérieur et de la Recherche Scientifique et Technique sous la tutelle du Ministère du plan et de l'aménagement du territoire. D'autres instituts sont mis en place en 1976: l'Institut de Recherche Agricole et Forestière, l'Institut de Recherche Zootechnique, l'Institut de Recherches sur les Techniques, l'Industrie et le Sous-sol et l'Institut des Sciences Humaines (ISH). En 1979, le Comité National de l'Homme et de la Biosphère et le Comité National de Développement des Technologies voient le jour avec pour rôle servir d'interface entre la science et la société. En 1984, les missions de recherches scientifique et technique sont élargies à l'enseignement supérieur dans le ministère de l'enseignement supérieur et de la recherche scientifique jusqu'en 1992. C'est l'âge d'or de la recherche camerounaise. En 1992, ce ministère est scindé entre le ministère de l'enseignement supérieur et celui de la recherche scientifique. Cette division, décriée aujourd'hui, semble faciliter à partir de 1993, en pleine crise économique et politique la création de six universités.

Le Ministère de la recherche scientifique et de l'innovation (MINRESI) à partir de 2004 est en charge de l'élaboration, de la mise en œuvre et de l'évaluation de la politique nationale de recherche scientifique et d'innovation. Pourtant d'autres acteurs au rang du Ministère de l'Enseignement Supérieur, le Ministère des Mines, de l'Industrie et du Développement Technologique ont des prérogatives concurrentielles en matière de recherche scientifique, de développement technologique et d'innovation.

Les conditions d'émergence de la recherche nationale camerounaise sont ainsi ancrées dans son hybridation avec les institutions internationales. Des années 1970 à 1990, les centres internationaux de recherche s'implantent au Cameroun (IITA, Icraf, Cifor, WorldFish, Adrao). Entre 1990 et 2000, les instituts de recherche français se restructurent dans le Centre de coopération Internationale en Recherche Agronomique pour le Développement (Cirad) puis la conversion de l'ORSTOM en Institut de Recherche pour le Développement (IRD). Le modèle d'innovation porté par ces institutions reste à l'époque polarisé par les conditions de réalisation du transfert technologique du nord vers le sud et du laboratoire scientifique vers les entrepreneurs utilisateurs. 


\section{La structure contemporaine du SNIR}

En 2015, la structure du SNIR Camerounais peut se décomposer en trois sous-systèmes au sein desquels se «distribue » l'activité de recherche (cf. Figure 1).

Figure 1 - Structure du Système National d'innovation et de Recherche Camerounais

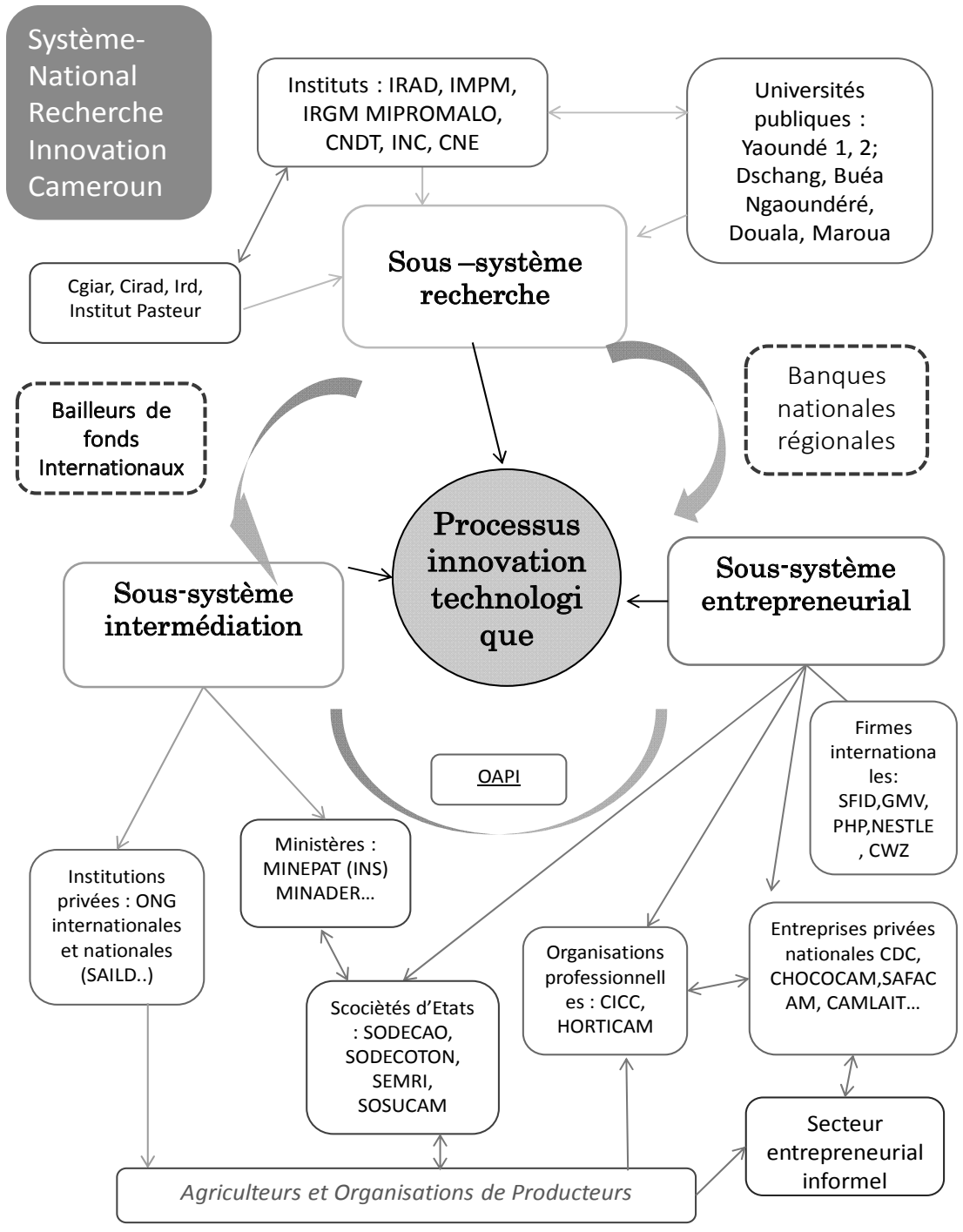

Source : Enquêtes à dires d'expert auteurs 2014 et 2016 
NB. Le premier sous-système est composé des institutions dédiées à la recherche scientifique. Il concerne le MINRESI qui coordonne les instituts thématiques de recherche et gouverne la politique de recherche nationale ${ }^{6}$. Sa réforme en 1991 est à l'origine de la dissolution de l'ISH et les créations du Comité National de Développement des Technologies $(\mathrm{CNDT})^{7}$, de la Mission de Promotion des Matériaux Locaux (MIPROMALO) et de l'Institut du Sahel. Les trois secteurs de concentration de la recherche en nombre de chercheurs sont polarisés par l'agriculture, la recherche minière et extractive et les sciences médicales (Tableau 2). Le deuxième ensemble est celui de la recherche universitaire gouvernée par le Ministère de l'enseignement supérieur. La carte universitaire initialement concentrée sur quatre ou cinq Universités d'État s'est diversifiée avec les universités de Buea, Bamenda et Maroua.

Dans ce sous-système de recherche, les universités concentrent de manière croissante les ressources humaines ( $89 \%$ des chercheurs nationaux) comme dans tous les pays en forte transition démographique où le nombre d'étudiants « explose » (UNESCO, 2015). Ayant pour mandat principal, la captation du savoir académique pour son transfert aux jeunes générations, l'Université a peu accès aux financements publics dédiés à la recherche. Les laboratoires expérimentaux financés pour partie par des coopérations internationales ne peuvent renouveler leurs équipements. Dans certains secteurs comme celui de l'agro-alimentaire, les capacités d'expérimentation nécessaires au transfert technologique de leurs résultats au secteur entrepreneurial sont en déclin. Les enseignants chercheurs tendent alors à privilégier des recherches fondamentales en fonction des opportunités de financements internationaux et des enjeux de carrière (Fofiri et al., 2015). Le risque de déconnexion de la recherche fondamentale universitaire par rapport aux besoins de recherche appliquée posés par le secteur entrepreneurial s'accroît. En soi, le concept de SNRI introduit la nécessité d'une recherche fondamentale problématisée par rapport à des enjeux prioritaires que posent les transformations sociétales.

Le deuxième sous-système est constitué par des institutions dédiées à l'intermédiation de l'innovation (Klerkx et al., 2012) : vulgarisation, formation, information, protection intellectuelle ou à son accompagnement. Elles sont distribuées au sein de différents ministères publics dont principalement l'agriculture, l'industrie, le Ministère du plan. Certaines sont institutionnalisées au sein de sociétés ou d'entreprises publiques (Sodecoton, Sodecao...) dotées d'une certaine autonomie. D'autres consistent en des instituts

6. Agence Nationale de Radio Protection (ANRP), Institut de Recherches Géologique et Minière (IRGM), Institut National de Cartographie (INC), Centre National de l'Éducation (CNE).

7. Le CNDT coordonne les activités pour transformer les résultats de recherche en innovations. 


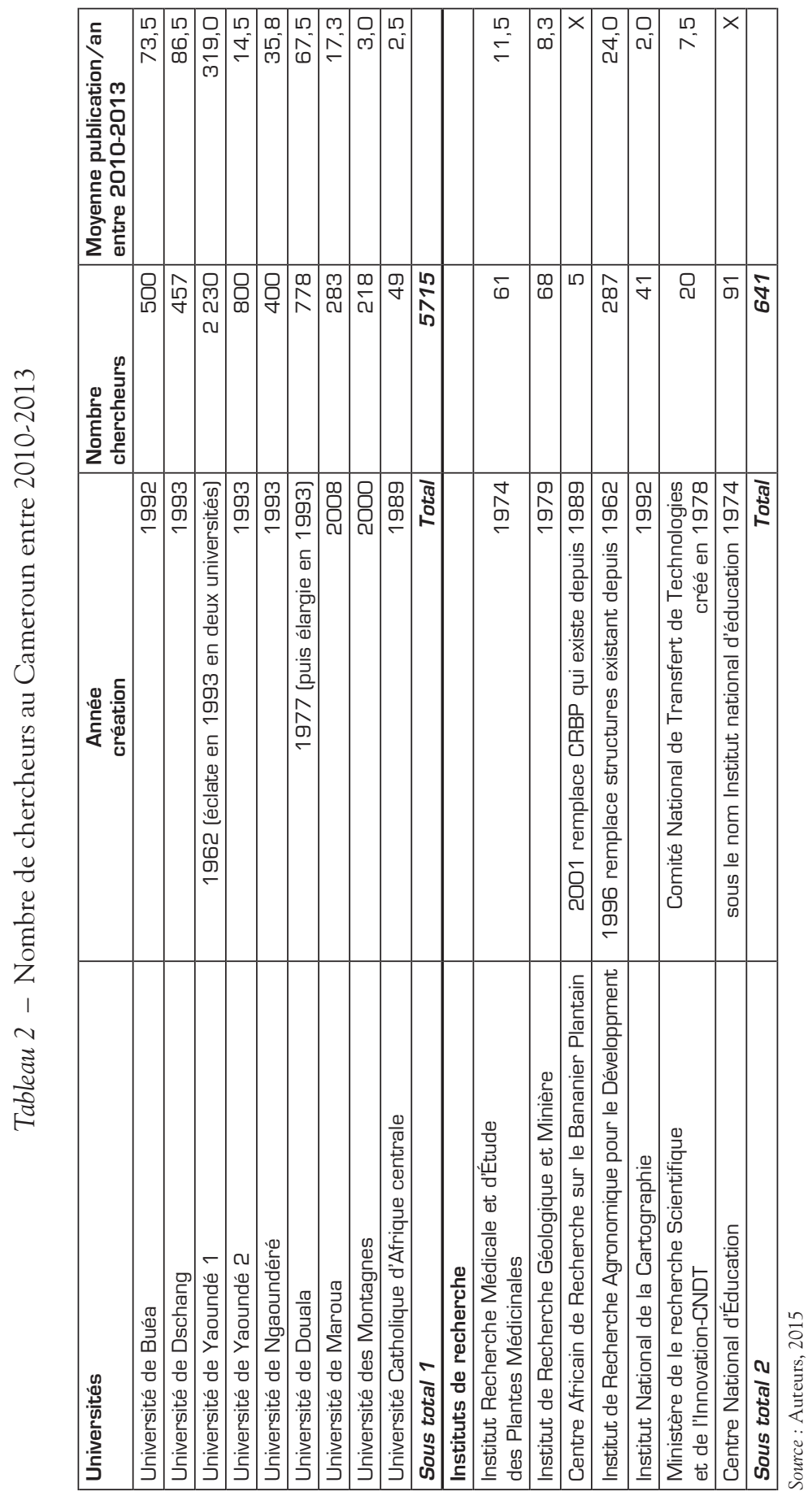


spécialisés à l'instar de l'Institut National de la Statistique (INS), principale source d'informations sur les entreprises. Ce sous-système se diversifie avec la croissance des institutions privées (ONG internationales ou nationales) qui ont émergé à la suite du désengagement public.

Le troisième sous-système concerne le secteur entrepreneurial qui au Cameroun se décompose entre un ensemble d'entreprises détenues par des nationaux (entreprises publiques et privées), et des entreprises privées étrangères qui assurent $54 \%$ de l'emploi permanent et sont concentrées dans l'exploitation des produits de base et la fourniture d'intrants (sylviculture, produits agricoles, énergie). Ce secteur d'entreprises officielles comptabilise environ 93000 emplois (permanents et temporaires) soit avec une estimation de la population active de 7,2 millions 1,3\% de la population active.

Le secteur informel incluant l'agriculture est peu mis en visibilité par les statistiques. En déduisant de la population active totale, la population active agricole (3,5 millions), l'emploi salarié industriel (93000 salariés), la population au chômage déclaré (13\% de la population active), le secteur informel procurerait de l'emploi à 3 millions de personnes. Est-il pris en compte dans les politiques de recherche et d'innovation actuelles?

\section{PRODUCTION SCIENTIFIQUE ET CONDITIONS DE SA MOBILISATION POUR L'INNOVATION}

\section{Une production scientifique croissante depuis 1991}

Lévolution de la production scientifique publiée par le Cameroun a été recensée en mobilisant différents moteurs de recherche bibliométrique ${ }^{8}$. Cet indicateur est un «proxy » de la production scientifique nationale. Il présente un biais car il exclut la production portant sur le Cameroun publiée par des chercheurs (camerounais ou internationaux) mais enregistrée dans le pays de localisation des auteurs. La production scientifique des chercheurs camerounais est en croissance continue depuis 2001 (cf. Figure 2).

Les universités d'État assurent sans surprise $58 \%$ de la production de connaissances scientifiques. En comparant les différentes universités, le taux des publications augmente quand le pourcentage d'étudiants par enseignant diminue. Les instituts du MINRESI contribuent pour $8 \%$ de la production et les centres internationaux de recherche basés au Cameroun pour $9 \%$ (cf. Figure 3).

8. Nous remercions M. C. Duchamps de la Délégation à l'Information Scientifique et Technique du Cirad pour son appui technique à la réalisation de l'interrogation bibliométrique. 
Figure 2 - Croissance de la production scientifique camerounaise depuis 1991

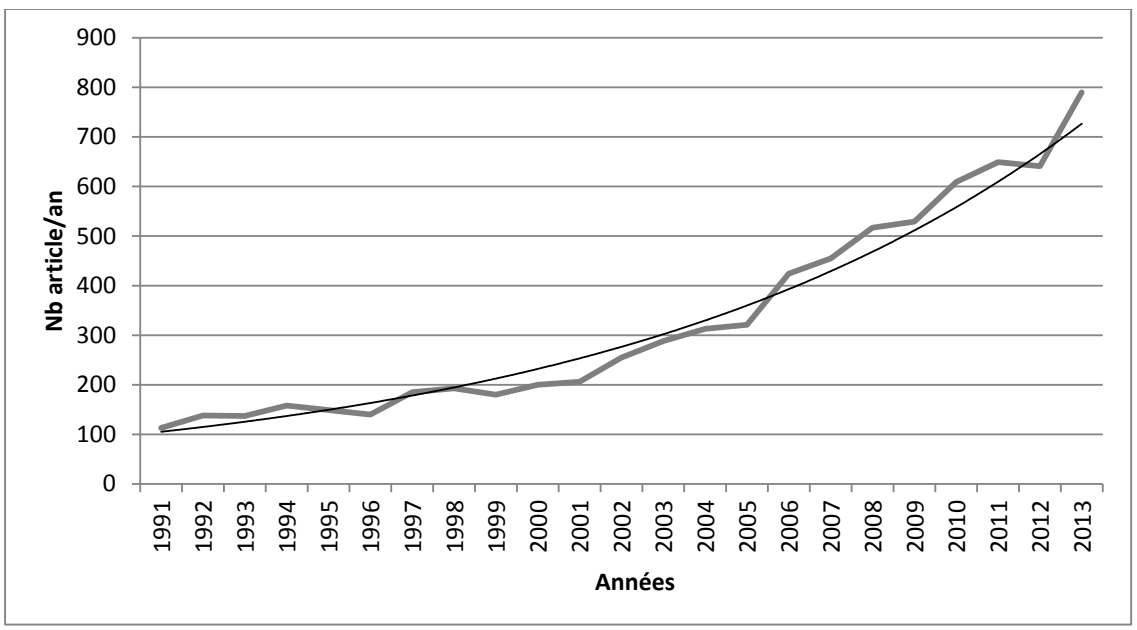

Source : Graphique - Auteurs et M. C. Duchamp (Cirad-Dist). Données - Web of ScienceTM Core Collection: Citation Indexes (SCI-Expanded, SSCI, A\&HCI). Produced by Thomson Reuters

Figure 3 - Répartition de la production scientifique (7 400 articles) identifiée entre 1991 et 2013 par organisme de recherche

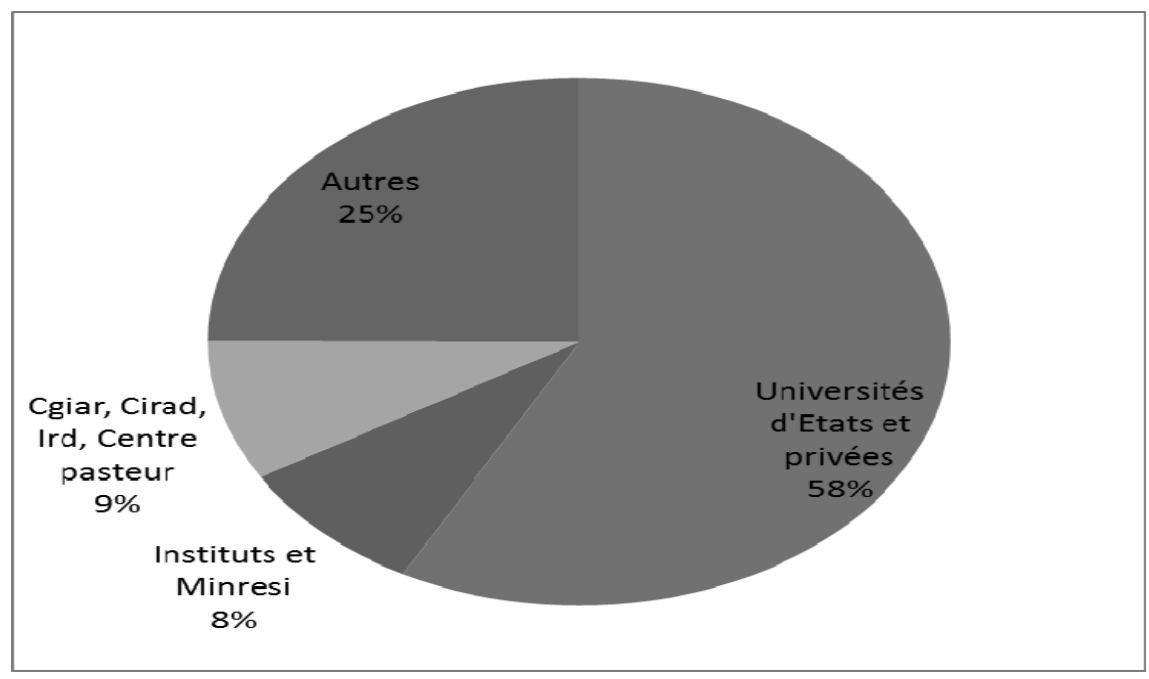

Source : Graphique : Auteurs et MC. Duchamp (Cirad-Dist). Données : Web of ScienceTM Core Collection: Citation Indexes (SCI-Expanded, SSCI, A\&HCI). Produced by Thomson Reuters. 
La croissance de la production scientifique du Cameroun est peu accessible aux acteurs du SNRI. Elle se concentre ces dix dernières années sur un nombre restreint de revues scientifiques référencées dans les bases internationales mais localement peu accessibles. Ainsi par exemple $48 \%$ des publications sont assurées par $7 \%$ des revues mais aucune institution de recherche n'a d'abonnement dans ces revues. A contrario, la plupart des revues nationales (académie des sciences, revue du CNDT) en format papier ne sont pas référencées ou ne sont pas numériquement accessibles.

\section{Évolution de la structure des publications scientifiques du Cameroun}

Une première interrogation regroupe sur l'échantillon total des publications (7 400) un sous-échantillon par grand secteur d'activité. La production scientifique ainsi liée au secteur agricole et alimentaire concentrerait sur cette base $30 \%$ de la production scientifique nationale. L'autre secteur de concentration majeure des productions scientifiques est celui de la santé et des recherches médicales (34\% des publications). Ces observations interrogent les relations qui peuvent être établies entre la recherche scientifique conduite au Cameroun et les besoins d'innovation du secteur entrepreneurial respectivement industriel et informel ou « artisanal ». Pour y répondre nous mobilisons une deuxième interrogation sur la structure intra sectorielle des publications scientifiques au sein de l'agriculture et de l'alimentation.

Jusqu'en 2003, le poids relatif des publications scientifiques dédiées aux cultures d'exportation, productions alimentaires et horticulture ne connaît pas de réelle différentiation. En revanche depuis 2003 la croissance des publications dédiées aux marchés internationaux (cacao, café, hévéa, palmier à huile, banane) tend à se démarquer de manière significative (cf. Figure 4). Il est à noter le cas particulier des filières horticoles qui ne sera pas analysé mais qui englobe les publications liées aux filières de bananes (export) et de plantains (marché intérieur). Le domaine des publications (Food Science and Technology) se référant au secteur de l'alimentation pour le marché intérieur central pour la croissance économique s'accroît mais avec environ $6 \%$ des publications, il reste relativement limité au regard du poids des publications polarisées par les marchés d'exportation.

Les tendances identifiées sur l'augmentation de la production scientifique liée au secteur agro-industriel d'exportation de produits de base et celui de l'agrofourniture (engrais chimiques, pesticides) interrogent sur les synergies existantes entre la production de connaissances scientifiques et les enjeux macro-économiques (sécurité alimentaire, environnement...) qui focalisent les processus d'innovations technologiques du secteur entrepreneurial. Ainsi 
la croissance des prix internationaux des produits agricoles de 2008 des produits agricoles parfois qualifiée de crise alimentaire (Meuriot et al., 2011) a renouvelé des objectifs productivistes d'accroissement de la production agricole sur le modèle d'intensification porté par les institutions ou les firmes des pays industriels.

Figure 4 - Structure de la production scientifique dans le secteur agricole et alimentaire

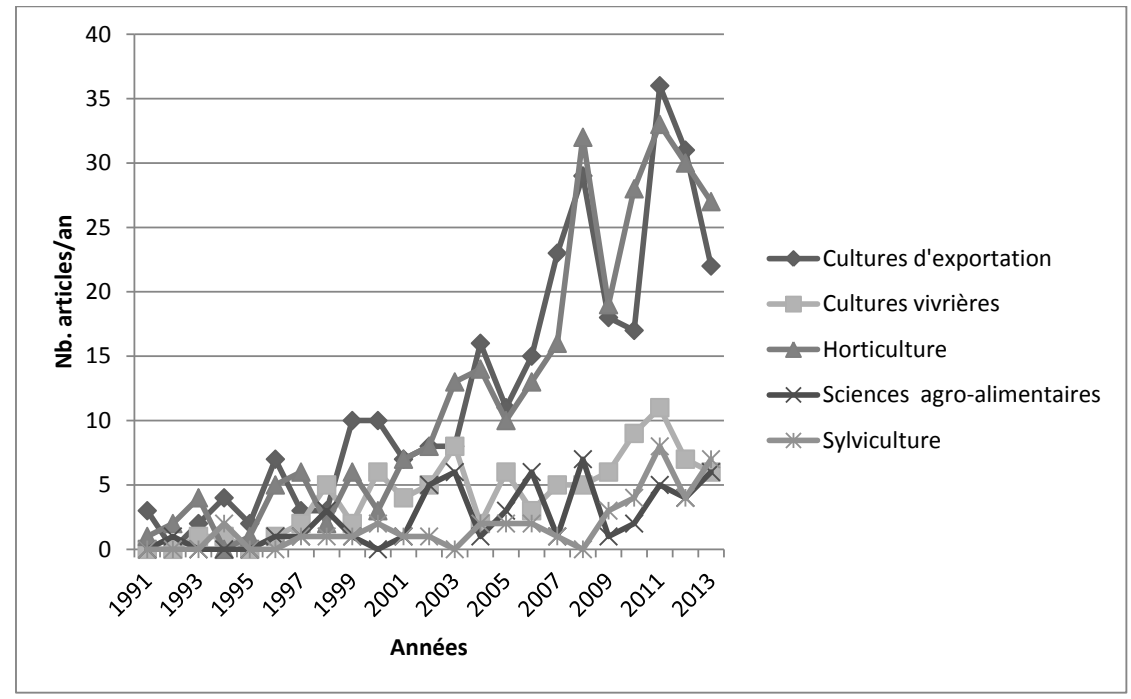

Source : Graphique - Auteurs et M. C. Duchamp (Cirad-Dist). Données - Web of ScienceTM Core Collection: Citation Indexes (SCI-Expanded, SSCI, A\&HCI). Produced by Thomson Reuters

Ce modèle génère-t-il à long terme des intégrations intersectorielles dans l'économie mondiale qui enclenchent des dynamiques de développement significatives? Ces évolutions sont-elles souhaitées par les pouvoirs publics ou bien résultent-elles de mécanismes plus complexes liés aux structures de financement de la recherche et de l'innovation?

\section{Le financement de la production scientifique : explication des tendances actuelles}

Les budgets alloués à la recherche scientifique et à l'innovation seraient en deçà de 0,02 \% du PIB au Cameroun (la norme moyenne dans les pays émergents est voisine de $1 \%$ du PIB : 1,07 \% en Malaisie, 0,87 en Afrique du sud). Ce financement mobilise deux sources principales (Bisson et al., 2014). Le financement public dédié à la recherche via le MINRESI depuis les plans 
d'ajustement structurel est en régression dans le temps long (sauf pour l'année 2013). Le MINESUP bénéficie, lui, du programme d'Appui au Système de l'Enseignement, Fonds de soutien à la recherche universitaire. D'autres fonds plus spécifiques sont également mobilisables : le Fonds d'Appui à la Recherche et à la Professionnalisation et le Fonds de Développement des filières Cacao et Café . La recherche bénéficie également de financements de coopération via l'Aide Publique au Développement, l'Union européenne, la Banque Mondiale (Fonds de Recherche sur Base Compétitive), et les challenges programmes des Cgiar ou les projets du CORAF. Ces financements sont soumis à plusieurs critiques (Stads, Beintema, 2015).

En ce qui concerne le financement public, il est parfois tributaire du rythme des rentrées fiscales d'où des instabilités ou des retards. En ce qui concerne le financement extérieur il est structuré par des appels à projets en général de 3 ans, alors que le temps d'aboutissement d'une recherche est plus long (20 ans par exemple pour investir une nouvelle variété en culture pérenne). Le «temps comptable » des appels à projets étant différent du "temps scientifique », les chercheurs sont conduits à consacrer une part de leur temps à "jongler » avec différents projets pour essayer de maintenir des activités de recherche stables. La phase d'obtention de résultats finalisés utilisables pour une transformation du secteur productif est en général la plus fragilisée. Les programmes de recherche sont en partie rythmés par les opportunités des appels d'offres internationaux ou ceux des grandes entreprises globalisées qui ne répondent pas forcément aux besoins prioritaires des acteurs socio-économiques du pays. En effet ces appels privilégient des indicateurs bibliométriques qui concentrent les sujets de recherche sur de la recherche fondamentale. Ils financent peu des infrastructures de recherche qui tendent à se dégrader et deviennent non fonctionnels. Les conséquences sont parfois un découragement des jeunes chercheurs nationaux des instituts qui privilégient des carrières administratives dans la fonction publique ou des migrations internationales (Afrique du sud, Canada, Europe).

\section{UTILISATION DE LA PRODUCTION SCIENTIFIQUE DANS LE SOUS-SYSTĖME ENTREPRENEURIAL ET D'INTERMÉDIATION}

\section{Structure de la croissance économique et du secteur industriel}

L'analyse des relations entre la production scientifique générée par le système national d'innovation et de ses performances dans la transformation 
du système productif, est confrontée à la difficulté de référencer les spécificités sectorielles. La croissance économique des cinq dernières années est principalement soutenue par le secteur tertiaire pour $47,8 \%$ du PIB en relation avec l'extension des activités dans les branches des transports, des télécommunications, du commerce, des banques et de l'hôtellerie. Le deuxième moteur de croissance est lié aux investissements dans le secteur secondaire : bâtiments et infrastructures dont une part est en relation avec les activités extractives (pétrole et gaz) et l'autre part à l'extension de l'urbanisation (croissance démographique).

L'urbanisation des modes de vie diversifie le modèle alimentaire et stimule le marché intérieur auquel le secteur agro-alimentaire a du mal à répondre. Les importations alimentaires par habitant augmentent rapidement. Ces difficultés sont paradoxales au regard des potentialités de l'agriculture camerounaise et de la concentration des investissements dans la recherche agronomique. Nous explorons ce paradoxe en caractérisant la demande de recherche du secteur agro-industriel. Ce dernier avec 318 entreprises pour 45000 emplois (incluant la branche agrochimie) assure près de 59 \% de l'emploi industriel du pays. Il connaît une structure très concentrée, ainsi 12 entreprises agro-industrielles (hévéa, sylviculture, palmier, sucre, banane) assurent $70 \%$ de l'emploi intra-sectoriel.

\section{La demande de recherche dans le secteur agricole et agro-alimentaire}

Les données macro-économiques mobilisables montrent que la productivité dans l'agriculture est faible sur le long terme. Ainsi l'indice de production agricole par la population active agricole (proxy de la productivité du travail) a baissé de $10 \%$ entre 1970 et 2006 (Fotso, Tsafack Nanfosso, 2014). Or selon l'enquête INS en 2009, 76 \% des entreprises du secteur de l'agriculture déclareraient utiliser des résultats de la recherche (11\% pour les autres secteurs). Cette contreperformance interpelle l'efficacité des investissements réalisés dans la recherche agronomique. Elle est contradictoire également avec les résultats d'expertises ponctuelles qui témoignent de success stories dans l'utilisation du matériel végétal, l'amélioration des itinéraires techniques (FAO, 1995). Cette contradiction serait liée aux caractéristiques macro-institutionnelles et aux structures de l'économie qui caractérisent les pays pauvres (Nyemeck, Nkamleu, 2006). En utilisant l'enquête Entreprises de l'Institut National de la Statistique nous précision ces structures dans le secteur industriel lié à l'agriculture et l'alimentation.

Les données disponibles classent les entreprises répertoriées en différentes branches d'activités. En regroupant ces branches en fonction du marché 
destinataire (marché international et intérieur), il est possible de comparer le poids relatif du secteur entrepreneurial industriel polarisé par l'exportation et celui pour l'approvisionnement d'un marché alimentaire en pleine croissance. Ce classement nourrit deux petites imperfections : l'industrie du sucre (marché intérieur) est comptabilisée dans l'agriculture d'exportation ; les entreprises de fruits et légumes (marchés mixtes) sont comptabilisées dans l'approvisionnement du marché intérieur. En posant pour hypothèse que ces deux imperfections se compensent, l'agriculture d'exportation et l'agriculture vivrière ont à peu près la même contribution du point de vue de l'emploi industriel. Mais avec un total d'emplois de 77828 identifiés dans le secteur industriel (dont 45973 dans l'agriculture industrielle et l'agroalimentaire) et une population active de 9 millions, ce secteur industriel emploie au Cameroun moins de $1 \%$ de la population active. L'essentiel de l'emploi est assuré par les services, l'agriculture non industrielle et le secteur entrepreneurial informel.

Dans le secteur industriel lié à l'agriculture vivrière pour le marché intérieur où la demande est en pleine croissance ( $c f$. Tableau 3 ), l'indicateur de la création d'emplois fait apparaître trois pôles. Le premier pôle concerne l'agriculture vivrière (plantain, manioc, igname...) et les productions animales. Le deuxième pôle est celui de la fabrication de produits à base de céréales : entreprises qui fabriquent des farines à partir de céréales importées du marché international (blé et maïs) ou qui distribuent les produits issus de cette transformation (boulangeries) intégrant la transformation de céréales locales : sorgho (Folefack et al., 2008). Le troisième pôle est constitué par les oléagineux avec la production d'huile de palme et l'approvisionnement en aliments de l'élevage dans un contexte d'accroissement de la consommation de viandes.

Les investissements des entreprises dans la recherche et l'expression de leurs besoins de recherche (Minkoua et al., 2016) différencient deux situations. Les entreprises privées qui incluent un ensemble de PME ou moyennes entreprises. Elles réalisent des recherches technologiques pour mettre au point de nouveaux produits ou adapter les technologies importées aux conditions locales. Ces investissements dans la recherche sont difficiles à identifier. Ils reposent sur des processus d'apprentissage par l'expérience et font peu l'objet de projets avec des scientifiques. La deuxième situation concerne des grandes entreprises agro-industrielles (CDC, SOCAPALM...) à gouvernance parfois semi-publique qui ont des activités de recherche finalisées par l'optimisation technico-économique de l'entreprise en partenariat avec des instituts nationaux comme l'IRAD ou parfois internationaux (CIRAD) : projets communs et mobilité de chercheurs. Les plus grandes (CDC) initient de manière nouvelle et encore rare des contrats de recherche avec les Universités. 
Tableau 3 - Structure en branches du secteur agro-industriel du Cameroun

\begin{tabular}{|c|c|c|c|c|c|}
\hline AGRO-INDUSTRIE D’EXPORTATION & & \begin{tabular}{|l|} 
Nbr. \\
Entrprise
\end{tabular} & $\%$ & $\begin{array}{l}\mathrm{Nr} . \\
\text { Emploi }\end{array}$ & $\%$ \\
\hline Agriculture industrielle et d'exportation & & 38 & 11 & 13427 & 29 \\
\hline Industrie du cacao, du café, du thé et du sucre & & 18 & 5 & 2251 & 5 \\
\hline Production de caoutchouc et fabrication d'articles & & 14 & 4 & 5829 & 13 \\
\hline \multirow[t]{2}{*}{ Sylviculture et exploitation forestière } & & 40 & 11 & 5053 & 11 \\
\hline & Total 1 & 110 & 31,25 & 26605 & 58 \\
\hline \multicolumn{6}{|l|}{ AGRO-INDUSTRIE-MARCHE INTÉRIEUR } \\
\hline Agriculture vivrière + élevage et pêche & & 27 & 8 & 6288 & 14 \\
\hline Fabrication de produits à base de céréales & & 145 & 41 & 3978 & 9 \\
\hline Industrie boisson & & 14 & 4 & 1526 & 3 \\
\hline Industrie d'oléagineux et d'aliments pour animaux & & 16 & 5 & 4870 & 11 \\
\hline $\begin{array}{l}\text { Industrie du lait, des fruits et légumes et autres } \\
\text { produits }\end{array}$ & & 16 & 5 & 1429 & 3 \\
\hline $\begin{array}{l}\text { Industrie chimiques et fabrication de produits } \\
\text { chimiques }\end{array}$ & & 20 & 6 & 1111 & 2 \\
\hline \multirow[t]{3}{*}{ Industries du tabac } & & 4 & 1 & 166 & O \\
\hline & Total 2 & 242 & 68,75 & 19368 & 42 \\
\hline & Total & 352 & 100 & 45973 & 100 \\
\hline
\end{tabular}

Source : Auteurs à partir de l'enquête Entreprise de l'Institut National de la Statistique 2009

Selon une expertise collective soutenue par le Groupement inter-patronal du Cameroun auprès de chercheurs et entrepreneurs (Fotso, Tsafack Nanfosso, 2014), l'inertie de la productivité dans l'agriculture serait une contrainte au développement de l'agro-industrie. Cette inertie s'expliquerait par la faible intensité capitalistique de l'agriculture en intrants industriels (engrais phytosanitaires, ...) et en capital fixe. Elle interroge les relations fonctionnelles au sein du SNI entre ce secteur de l'agrofourniture, la recherche publique nationale et les besoins des agriculteurs. Pour tester ces relations, les résultats de deux ateliers de concertation auprès des entreprises du sous-secteur phytosanitaire et semencier ont été mobilisés. Les opérateurs invités y étaient interrogés sur leurs perceptions des mécanismes de transfert des résultats de recherche et sur les facteurs majeurs de blocages à l'amélioration de cette transférabilité. Une synthèse des résultats reclasse les contraintes majeures selon qu'elles concernent plutôt des questions de « recherche » ou « d'innovation ».

\section{Les contraintes pour l'usage des connaissances scientifiques dans l'agrofourniture}

L'échantillon consulté portait sur les 15 principales firmes impliquées dans l'agrofourniture (phytosanitaire et semencier) présentent au 
Cameroun'. La représentativité de cet échantillon est partielle car elle n'inclut pas les petites entreprises de production de plants. La plupart des entreprises fabriquent ou utilisent des intrants mis au point par des laboratoires de recherche ou des firmes " globalisées » rattachées à des groupes mais avec peu d'activités de production au Cameroun. Certaines des entreprises consultées soulignent néanmoins leur intérêt pour investir sur des intrants valorisant des ressources locales (bio pesticides à partir de feuilles de neem...). Toutes les firmes citent le manque d'interaction avec la recherche nationale. Elles expriment des besoins de recherche pour évaluer l'écotoxicité sur la santé et l'environnement des produits nouveaux ou bien pour mieux contrôler la fraude sur les produits phytosanitaires importés. Les principaux facteurs limitants dans l'usage des résultats de la recherche camerounaise portent sur les éléments prioritaires suivants.

Au niveau de la politique de recherche, il n'y a pas d'information disponible sur (i) les résultats de la recherche : 3000 brevets, on en connaît et utilise aucun »; (ii) les programmes notamment en chimie ; (iii) les compétences mobilisables; (iv) les laboratoires fonctionnels. Il n'y a aucun mécanisme d'appui des interactions pour produire, utiliser des « connaissances collectives " sur les conditions d'usages des inventions des chercheurs ou encore tester des risques sanitaires, environnementaux ou d'écotoxicité.

Au niveau de la politique d'innovation, il n'y a pas d'incitation pour investir sur des innovations à fort potentiel de croissance de marché mais qui comportent des risques de rentabilité. La taille limitée du marché intérieur ou des marchés de niches sur de nouveaux intrants, bloque des investissements locaux dans un contexte de mobilisation du financement bancaire défaillant. Les quelques conventions avec des grandes entreprises répertoriées sur la mise en œuvre de programmes/projets (formation, fourniture ponctuelle de matériel végétal, etc.) sont très ponctuelles. Le secteur entrepreneurial agro-chimique véhicule plutôt une recherche adaptative pour fixer les conditions d'usage d'intrants industriels mis au point dans des firmes mondialisées. Il est au centre du modèle d'innovation linéaire visant à homogénéiser les conditions sociales de production au regard d'objectifs industriels de standardisation de l'offre agricole pour approvisionner les marchés internationaux ou des unités de transformation agro-industrielle (huile, sucre...). En revanche, ce secteur accompagne très peu les besoins d'innovation de l'agriculture vivrière qui approvisionne les marchés alimentaires diversifiés et localisés au regard des attentes des consommateurs (Dury et al., 2002) et plus largement celui des dynamiques agro-alimentaires du secteur informel.

9. Duponds Nemour, Sygenta, Fimex, Semagri, LDC. 


\section{Contraintes à l'usage des connaissances scientifiques par les organisations professionnelles de l'intermédiation}

Le désengagement de l'État des politiques publiques dans les années 1980 à 2000 en relation avec les programmes d'ajustement structurel a stimulé l'émergence d'une multitude de nouveaux acteurs: Organisations Non Gouvernementales, Organisations de Producteurs engagés dans une diversité d'activités: animation rurale, vulgarisation, commercialisation agricole, expérimentation technique. Un certain nombre d'entre eux polarisent s activités sur le renforcement des capacités. Ils deviennent des structures d'intermédiation des résultats de la recherche en agriculture. Ces acteurs peuvent être classés dans la typologie suivante.

a) Les ONG internationales telles que le SAILD et le CIPCRE qui font partie des plus importantes dans l'agriculture. Leurs actions de formations portent sur l'animation à l'autopromotion, la protection de l'environnement et l'accompagnement des activités économiques des organisations paysannes.

b) Des réseaux d'ONG nationales comme le Zenü Network qui regroupe plusieurs ONG nationales dans divers secteurs (décentralisation, santé, agriculture, environnement, ...) basées dans la région de l'Ouest. Ces ONG ont été créées dans la plupart des cas pour sensibiliser des producteurs à se regrouper en organisations paysannes agricoles sous forme de Groupes d'Initiative Commune (GIC) et de sociétés coopératives.

c) Les organisations interprofessionnelles. La première dans le secteur agricole est le CICC mis en place par l'État en 1991. Plusieurs autres organisations interprofessionnelles comme l'IPAVIC dans le secteur avicole, RHORTICAM dans le secteur horticole ont vu le jour ces dernières années.

d) Les organisations de producteurs et leurs regroupements : aux niveaux national, régional et local. En 2011, le Cameroun comptait ainsi 121730 organisations paysannes agricoles légalisées par les services du registre Coop/GIC. Ces organisations paysannes sont enregistrées comme coopératives ou GIC (Fongang, 2012).

L'organisation de deux ateliers d'enquêtes participatives a permis de consulter un échantillon d'organisations constitué de 15 Organisations Professionnelles $(\mathrm{OP})$ et institutions de l'intermédiation. Ces ateliers ont caractérisé les domaines de collaboration avec la recherche agricole sur deux axes complémentaires :

- la vulgarisation des résultats et inventions de la recherche : itinéraires techniques de production, matériel végétal (maïs, papaye, manioc, patate douce, etc.) dans une logique de transfert technique et ; 
- les collaborations avec les chercheurs pour élaborer à partir de ressources locales (cognitives, expérimentales, naturelles...) de nouveaux intrants, produits, pratiques; réaliser des formations techniques, mettre au point des fiches techniques et des essais en milieu rural.

Les relations entre les $\mathrm{OP}$ et les universités sont apparues limitées à l'accueil des étudiants en stage académique. La faible reconnaissance par les universités du caractère formateur des stages et du statut de "partenaire » des institutions ou entreprises d'accueil des stages est identifiée comme un facteur limitant à la densification de ces interactions.

Les partenariats entre les organisations d'intermédiation et les instituts de recherche sont soulignés comme peu institutionnalisés. Ils sont essentiellement portés par des relations interindividuelles. Si l'ensemble des opérateurs souligne l'existence passée de success stories dans l'usage des propositions de recherche dans les années 1970 et 1980, la perte de confiance dans les conditions de validation expérimentale diminue cet usage. Elle s'explique par les items repérés suivants :

- Le manque d'adaptation des thèmes de recherche aux besoins du monde socioéconomique. Verbatim 1: «Les chercheurs développent les thèmes en fonction des opportunités qu'ils ont et non sur la base des problèmes du monde socio-économique ».

- Le manque de moyens pour conduire des activités expérimentales en partenariat avec le secteur entrepreneurial, notamment les agriculteurs et les entreprises du secteur agrochimique. La plupart des structures de recherche disposent d'équipements vétustes et peu adaptés pour répondre aux besoins actuels des entreprises.

- Le manque de stratégie de valorisation. La recherche n'accorde pas d'attention à la manière dont ses résultats seront mis à la connaissance du reste de la société. Il s'ensuit l'absence de dispositifs de communication et de valorisation des résultats de la recherche (internet, radio, etc.). Verbatim 2: "Regardez l'IRAD qui n'a aucun dispositif de diffusion des innovations. Leur site internet est vide de ce point de vue, vous n'y voyez rien ».

Par ailleurs les complémentarités d'actions avec les activités de vulgarisation publique (semences améliorées, nouvelles techniques de production, etc.) manquent de mise en cohérence politique (Fofiri et al., 2016).

En fonction de ces résultats la critique sur la faible connectivité des résultats de recherche aux besoins portés par les structures d'intermédiations publiques ou privées peut se spécifier selon deux trajectoires d'innovation qui ne sont pas forcément convergentes. La première renvoie aux défaillances des structures de diffusion pour rendre les résultats de la recherche utilisables. La faible mise en usage des résultats de la recherche y est alors 
expliquée par la défaillance des structures de transfert technologique et non le manque d'adaptation des résultats aux dynamiques d'innovation portées par les réalités entrepreneuriales. La deuxième trajectoire renvoie à l'émergence d'un modèle d'innovation ouverte dans lequel la recherche hybride les bases de connaissances scientifiques et techniques pour accélérer les processus de transformation de l'agriculture à partir des ressources productives localisées. Plus en phase avec un processus d'écologisation de la fonction de production agricole, cette trajectoire se construit par la capacité à synchroniser les dimensions organisationnelles (réseaux d'acteurs) institutionnelles (normes, valeurs, incitations publiques) et technologiques de l'innovation en agriculture (Temple et al., 2015).

\section{CONCLUSION}

Les travaux sur les Systèmes Nationaux d'Innovation et de Recherche soulignent en quoi les interactions entre les investissements publics dans la recherche scientifique et le secteur de l'entreprise sont des éléments structurant de l'innovation technologique dans l'agriculture et l'alimentation. Ces relations sont centrées sur deux axes.

Le premier axe porte sur la complémentarité entre les différentes ressources que génère la recherche: nouvelles connaissances, compétences, «nouveautés» de différentes natures: physiques (produits, intrants), concepts (applications numériques), institutions (normes, habitudes), ou organisations assurant les coordinations entre les agents économiques. Le deuxième axe porte sur les capacités de mobilisation des ressources générées par la recherche par les dynamiques entrepreneuriales liées à l'évolution de la structure du système productif.

Les résultats mis en exergue au Cameroun soulignent que les relations entre les sous-systèmes qui forment la structure d'un potentiel système national de recherche et d'innovation ne sont ni régulières, ni stables. Au regard de ces observations ce système national de recherche et d'innovation n'existerait pas en tant « qu'objet » que peut révéler l'analyse. Il existe en tant que « projet » qui pourrait être construit par la mise en cohérence de politiques de recherche et d'innovation en cours. Ce projet est contraint par une fragilité institutionnelle qui renvoie à l'amélioration de la gouvernance globale. Cette gouvernance reste encore parfois trop marquée par une faible différenciation de l'espace public et de l'espace privé. L'un des ressorts en est l'inclinaison néo-patrimoniale du pouvoir qui fonde parfois la gestion des affaires publiques comme élément du patrimoine privé à l'encontre des règles formelles qui peuvent être tactiquement utilisées à des fins personnelles. Elle 
peut constituer un frein à l'implémentation efficace des politiques publiques en matière de recherche et d'innovation.

Le secteur agricole et alimentaire structure le système productif actuel de l'économie camerounaise. Il présente d'importantes marges de croissance pour l'approvisionnement du marché intérieur au regard de l'accroissement rapide de la population urbaine et rurale en relation avec la transition sociodémographique.

Dans ce secteur, le système national de recherche issu d'investissements publics de long terme dispose de potentialités à générer des connaissances technologiques qui répondent mieux aux besoins d'accompagnement (apprentissages, expérimentations) et d'innovation technologique de l'entrepreneuriat. La relation entre la recherche et les dynamiques entrepreneuriales qui portent l'innovation ne peut se cantonner au seul secteur industriel au regard de son poids relatif dans l'emploi.

Il apparaît nécessaire au-delà du transfert de technologies élaboré dans d'autres contextes de développement (Lundval, 2014) de renforcer les politiques d'innovation et de recherche pour mieux identifier et lever les verrous technologiques que rencontre l'entrepreneuriat particulièrement dans le secteur agro-artisanale parfois informel. Ce secteur en effet constitué de moyennes et petites entreprises de transformation concentre la plus grande partie de la population dans l'approvisionnement du marché intérieur. L'implication de la recherche dans la valorisation des ressources locales: biodiversité, savoir-faire tant pour fabriquer des intrants que des produits alimentaires est un axe structurant des synergies entre politique de recherche et d'innovation. Elle interroge une certaine inertie technologique au regard d'une production scientifique qui privilégie souvent des thématiques d'approvisionnement des marchés internationaux et d'intensification industrielle de l'agriculture à partir d'intrants produits par des firmes globalisées (Hall, 2005). Cette «inertie technologique » est en réalité hétérogène au regard des dynamiques d'innovations réelles.

Dans le diagnostic proposé, une contribution de la recherche à l'innovation a été peu intégrée. Elle concerne la fonction de formation par la recherche. Elle renvoie à l'implication des universités dans la formation des capacités intellectuelles des nouvelles générations à se saisir des connaissances scientifiques et technologiques que propose la globalisation rapide de l'économie de la connaissance permise par la révolution numérique (Kamdem, 2014). 


\section{BIBLIOGRAPHIE}

AFRICAN UNION NEW PARTNERSHIP FOR AFRICA'S DEVELOPMENT (2014), African Innovation Outlook, Pretoria, AU-NEPAD.

ARVANITIS, R., WAAST, R., GAILLARD, J. (2000), Science in Africa: A Bibliometric Panorama Using Database, Scienciometrics, 47, 457-473.

BANQUE MONDIALE (2015), A Decade of Development in Sub-Saharan African Science Technology Engineering $\mathcal{E}$ Mahematic Research, Washington, World Bank and Elsevier.

BISSON, P., ALAMI, S., TEMPLE, L. (2014), Etude sur l'économie de la recherche au Cameroun, Programme d'Appui à la Recherche. Ministère de la recherche scientifique et de l'innovation, Yaoundé, Cameroun Cirad.

BORNMAN, L. (2013), What is Societal Impact of Research and How Can it be Assessed? A Literature Survey, Journal of the American Society for Information Science and Technology, 64(2), 217-233.

CARLSON, B. (2006), Internationalization of Innovation Systems: A Survey of the Literature, Research Policy, 35, 56-67.

CASADELLA, V., LIU, Z., UZUNIDIS, D. (2015), Développement économique et capacités d'innovation dans la mondialisation, Londres, ISTE.

DURY, S., BRICAS, N., TCHANGO TCHANGO, J., TEMPLE, L., BIKOI, A. (2002), The Determinants of Urban Plantain Consumption in Cameroon, Food Quality and Preference, 13(2), 81-88.

FAO-MINREST, (1995), Le système national de recherche agricole du Cameroun. Analyse et proposition de stratégie pour le long terme, Rome, FAO.

FOFIRI NZOSSIE, E. J., TEMPLE, L., NDJOUENKEU, R. (2015), La contribution de la recherche universitaire à la formation d'un système sectoriel d'innovation agro-alimentaire au Cameroun, Innovations, 47, 55-77.

FOFIRI NZOSSIE, E. J., NDAME, J. P., TEMPLE L., SIMEU KAMDEM, M., (2016). L'innovation agricole dans la zone soudano-sahélienne du Cameroun: acteurs et politique d'intervention, in Ngo Balepa, A.S.F., Moupou, M., Mougoue, B., Nji Fogwe, Z., Tchawa, P., Pour une géographie rurale de l'action, Edition Clé Yaoundé, 411-425.

FONGANG FOUEPE, G. H. (2012), Les organisations de producteurs agricoles en Afrique de l'Ouest et du Centre : diversité, dynamique, rôle des politiques publiques : le cas du Cameroun, Paris, Institut de Recherches et d'Applications des Méthodes de développement.

FOLEFACK, D. P., NJOMAHA, C., DJOULDÉ, D. R. (2008), Diagnostic du système de production et de commercialisation du jus d'oseille de Guinée dans la ville de Maroua, Tropicultura, 26(4), 211-215.

FOTSO, A., TSAFACK NANFOSSO, R. (2014), 100 propositions pour l'émergence du Cameroun, Yaoundé, CREG.

FREEMAN, C. (2002), Continental, National and Sub-national Innovation Systems: Complementarity and Economic Growth, Research Policy, 31(2), 191-211.

GAILlARD, J. (2003), Overcoming the Scientific Generation Gap in Africa: An Urgent Priority, Interdisciplinary Science Reviews, 28(1), 15-25.

GAILlARD, J. M., HASSAM, A., WAAST, R. (2005), Africa in Unesco World Science Report, Paris, UNESCO Publishing. 
GALLAUD, D. (2014), Les systèmes nationaux d'innovation approches théoriques, in Principes d'économie de l'innovation, Boutillier, S., Forest, J., Gallaud, D., Laperche, B., Tanguy, C., Temri, L. (dir.), Business et Innovation, Bruxelles, Peter Lang, 377-402.

GEELS, F. W. (2004), From Sectoral Systems of Innovation to Socio-technical Systems: Insights about Dynamics and Change from Sociology and Institutional Theory, Research Policy, 33(6-7), 897-920.

HALL, A. (2005), Capacity Development for Agricultural Biotechnology in Developing Countries: An Innovation Systems View of What It is and How to Develop It, Journal of International Development, 17(5), 611-630.

KAMDEM, M. S., (2014), L'Université africaine et sa contribution au développement local. L'exemple du Cameroun, Yaoundé, Karthala.

KLERKX, L., MIERLO (VAN), B., LEEUWIS, C. (2012), Évolution of Systems Approaches to Agricultural Innovation: Concepts, Analysis and Interventions, in Darnhofer, I., Gibbon, D., Dedieu, B. (eds), Farming Systems Research into the 21st Century, New York, Springer.

LAREDO, P., MUSTAR, P. (2003), Politique publique de recherche et d'innovation, in Mustar P., Penan H. (dir.), Encyclopédie de l'innovation, Paris, Economica.

LOSEGO, P. (2008), Quelle politique de la science pour un pays intermédiaire? Le cas des sciences de la nature en Afrique du Sud (1945-2006), Revue d'Anthropologie des Connaissances, 2, 361-390.

LUNDVALL, B. A., LEMAPAGES, R. (2014), Growth and Structural Change in Africa: Development Strategies for the Learning Economy, African Journal of Science, Technology, Innovation and Development, 6(5), 455-466.

MACHIKOU, N. (2014), La sédimentation d'une communauté de connaissance au cœur de l'action publique en Afrique. Un nouveau souffle pour les politiques publiques?, in Hourquebie, F. (dir.), La doctrine en Afrique noire francophone, Bruxelles, Bruylant.

MALERBA, F. (2002), Sectoral Systems of Innovation and Production, Research Policy, 31, 247-264.

MAREDIA, M. K., RAITZER, D. A. (2010), Estimating Overall Returns to International Agricultural Research in Africa through Benefit-Cost Analysis: A Best-evidence Approach, Agricultural Economics, 41(1), 81-100.

MEURIOT, V., TEMPLE, L., MADI, A. (2011), Faible transmission des prix internationaux aux marchés domestiques: le poids des habitudes alimentaires au Cameroun, Economie Appliquée, 3, 59-84.

MINKOUA, J. R., TEMPLE, L. (2016), Impact of Research on Development in Cameroon: Convergence between Supply and Research Needs in the Food Sector, Communication 21 Conférence on Science Technology and Indicators, Valencia, Book of Proceedings, 366-374.

MINSAT, A., SIMPASA, A., LUSIGI, A., LOSCH, B. (2015), African Economic Outlook: Thinking Regional to Foster Africa's Structural Transformation, Great Insights, 4(4), 4-7.

MYTELKA, L. K.(2003), The Dynamics of Catching up: the Relevance of an Innovation System Approach in Africa, in Muchie, M., Gammeltoft, P., Lundvall, B. A. (eds), Putting Africa First: The Making of African Innovation Systems, Aalborg University Press, Danemark, 29-43.

NYEMECK, B. J., NKAMLEU, G. B. (2006), Potentiel de productivité et efficacité technique du secteur agricole en Afrique, Canadian Journal of Agricultural Economics, 54(3), 361-377. 
STADS, G. J., BEINTEMA, N. (2015), Agricultural R\&D Expenditure in Africa: An Analysis of Growth and Volatility, European Journal of Development Research, 27, 391-406. TEMPLE, L., TOUZARD, J. M., BOYER, J., REQUIER DESJARDINS, D. (2015), Comparaison des trajectoires d'innovation pour la sécurisation alimentaire des pays du Sud, Biotechnol. Agron. Soc. Environ. 19(1), 53-61, http://popups.ulg.ac.be/1780-4507/index.php?id=11824.

TEMPLE, L., BIENABE, E., BARRET, D., SAINT-MARTIN, G. (2016), Methods for Assessing the Impact of Research on Innovation and Development in the Agriculture and Food Sectors, African Journal of Science, Technology, Innovation and Development, 399-410, http://dx.doi.org/10.1080/20421338.2016.1219484

TOUZARD, J. M., TEMPLE, L., FAURE, G., TRIOMPHE, B., (2015), Innovation Systems and Knowledge Communities in the Agriculture and Agrifood Sector, Journal of Innovation Economics E⿱ Management, 17(2), 116-140.

UZUNIDIS, D., LAPERCHE, B. (2011), The New Mercantilism and the Crisis of the Global Knowledge Economy, Journal of the Knowledge Economy, 2(3), 373-392.

UNESCO (2015), Higher Education, Research and Innovation: Changing Dynamics, Meek, V. L., Teichler, U., Kearney, M. L. (eds), Report on the UNESCO Forum on Higher Education, Research and Knowledge 2001-2009, International Centre for Higher Education Research Kassel (INCHER-Kassel).

WAAST, R., GAILLARD, J. (2001), La science en Afrique à l'aube du 21 ème siècle, Rapport final Commission Européenne, DG XII, Subvention Nº 980149 800, France, Ministère des Affaires Etrangères. 